\title{
Philosophiques
}

\section{Descartes : discours et méthode}

\section{Daniel Dumouchel}

Volume 23, numéro 2, automne 1996

URI : https://id.erudit.org/iderudit/027404ar

DOI : https://doi.org/10.7202/027404ar

Aller au sommaire du numéro

Éditeur(s)

Société de philosophie du Québec

ISSN

0316-2923 (imprimé)

1492-1391 (numérique)

Découvrir la revue

Citer cet article

Dumouchel, D. (1996). Descartes : discours et méthode. Philosophiques, 23(2),

373-387. https://doi.org/10.7202/027404ar

Ce document est protégé par la loi sur le droit d'auteur. L'utilisation des services d'Érudit (y compris la reproduction) est assujettie à sa politique d'utilisation que vous pouvez consulter en ligne.

https://apropos.erudit.org/fr/usagers/politique-dutilisation/ 


\title{
DESCARTES : DISCOURS EI MÉTHODE
}

\author{
PAR \\ Daniel Dumouchel
}

Il s'agira ici d'analyser les rapports qu'entretiennent chez Descartes la "méthode " et le "discours". En dissociant, dans l'intitulé de ce texte, le "discours " de la "méthode ", je tiens à souligner ce que l'on pourrait appeler la dimension "discursive" de la méthode cartésienne, c'est-à-dire le fait que la "méthode "cartésienne, fondée sur le paradigme de la certitude mathématique, et par conséquent sur une conception anhistorique et auto-instauratrice de la raison et de la "subjectivité ", repose sur des formes de discursivité ou de narrativité spécifiques. On obtient ainsi un sens un peu différent de ce que signifie "discours de la méthode ": il ne s'agit plus alors tout à fait d'un discours tenu sur la méthode, mais plutôt, en interprétant autrement le génitif, du discours de la méthode elle-méme, c'est-àdire des structures discursives dont cette mèthode, dans son instauration philosophique, ne peut ètre entièrement séparée.

En effet, la philosophie cartésienne - le discours cartésien - fait un usage large et diversifiè de toutes les possibilités discursives et narratives de la littérature et de la rhétorique dans la philosophie. Jamais, depuis Platon, un philosophe de l'esprit de géométrie n'aura tant usé de l'esprit de finesse. Bien que l'on puisse considérer la pensée cartésienne comme un exemple privilégié du paradigme classique en philosophie, il est aisé de remarquer à quel point Descartes utilise cela même qu'il prétend évacuer et discréditer. Ainsi, n'est-il pas étonnant que ce " classique " épris de clarté et d'ordre, qui fut la source d'inspiration des esthétiques et des rhétoriques classiques les plus contraignantes, telles que l'Art poétique de Boileau ou la Lettre à l'Académie sur les discours de Fénelon, ait fait un usage si abondant, et pendant la quasi-totalité de son parcours philosophique, de toutes les stratégies et de tous les artifices littéraires et rhétoriques du "baroque", comme l'hyperbole, l'allégorie, la fiction, la fable, la méditation, en plus d'en déployer les thèmes de prédilection : le doute, la déhiscence de la veille et du rêve, de la raison et de la folie, l'opacité de la représentation, les masques, le double (sous la forme du mauvais géniel ? Que penser de cet apótre de la raison pure et du savoir axiomatique qui avoue ne s'avancer que "masqué "(larvatus prodeo, comme l'annoncent les Préambules ${ }^{1}$ ), et qui répétera à plusieurs reprises par la suite qu'il préfère rester caché derrière son

1. Les textes de Descartes seront cités selon l'édition qu'a donnée F. Alquié des OEwres philosophiques, 3 tomes, Paris, Bordas ("Classiques Garnier "). 1988-1989 (citée désormais: O.P. suivi du numéro de volume et de la page). Lorsque c'est possible, j'indiquerai la référence dans l'édition des CEures de Descartes, publiée par C. Adam et P. Tannery. Paris, Léopold Cerf, 1897-1913 (citée : A.T., suivi du numéro de volume et de la page). Le texte des Préambules se trouve en A.T. X. 212 ; O.P. I, 45. 
œuvre sans dévoiler ses intentions ${ }^{2}$ ? Que penser encore de ce philosophe dont le projet intellectuel semble tout entier renfermé dans une allégorie multiple qui lui fut révélée dès le départ par des rêves - c'est le texte des Olympiques, que le Discours de la méthode raconte sous une forme plus sereine et plus réfléchie dans l'épisode du poèle - et dont la philosophie subséquente pourrait n'étre que le décryptage progressif? Il y a donc bel et bien une "écriture" cartésienne. Plus qu'un "style", donc, ce qu'il faut bien appeler le " discours " cartésien mérite d'ètre considéré comme une dimension consubstantielle de la pensée philosophique de Descartes.

Il conviendra donc de partir du fait qu'il y a chez Descartes non seulement un "discours de la méthode ", par où la "méthode " nouvelle qui doit rendre possible la réforme des sciences et de la philosophie est présentée, mais qu'il y a également une " méthode du discours". par le biais duquel cette méthode est en même temps légitimée. Nous sommes autorisés, en étudiant les principaux textes cartésiens, et tout particulièrement le Discours de la méthode et les Méditations de philosophie première, à parler de la méthode comme discours, mais également du discours comme méthode. Si la méthode, au sens propre, est une manière de cheminer et de "conduire sa pensée ", comme le répète si souvent Descartes ${ }^{3}$, la " discursivité " du texte cartésien nous ouvre à la façon dont la pensée philosophique de Descartes a été conduite, ou du moins à la façon dont elle a organisé sa propre présentation et son auto-légitimation. C'est là d'ailleurs l'un des thèmes qui me retiendra le plus : la discursivité ou la narrativité du Discours et des Méditations sont liées directement à la tâche philosophique de la justification de la nouvelle conception de la subjectivité à laquelle doit nous conduire la philosophie cartésiennet.

2. "Bene vixit, bene qui latuit" : lettre à Mersenne, avril 1634 (O.P. I, 495). Alquié, en faisant remarquer que le vers est d'Ovide (Tristes), traduit : "Il a bien vécu, celui qui a vécu caché ".

3. Et il y insiste déjà dans le sous-titre du Discours : "Pour bien conduire sa raison et chercher la vérité dans les sciences". Mais le thème est un véritable leitmotiv : la première partie du Discours prétend représenter la vie de Descartes "comme en un tableau ", afin de "faire voir [...] les chemins [qu'il] a suivis" (A.T. VI, 4-5; O.P. I, 570) : Descartes précise que son dessein n'est pas d'enseigner "la méthode que chacun doit suivre pour bien conduire sa raison", mais de faire voir comment il a "tãché de conduire "la sienne (A.T. VI, 5; O.P. I, 571) ; il est finalement question d'une résolution " d'employer toutes les forces de [son] esprit à choisir les chemins "qu'il devait suivre (A.T. VI, 10; O.P. I, 578), Le theme de la conduite de la pensée est alors explicitement reliè à la dimension pratique de la recherche philosophique et au désir de "marcher avec assurance en cette vie " (A.T. VI, 10 ;O.P. I, 577) et de bien "conduire sa vie "(Discours II, A.T. VI, 14 ; O.P. I, 581), qui semble constituer la disposition d'esprit la plus originaire de Descartes. Les Règles pour la direction de l'esprit précisaient déjà que " l'objet des études doit être de diriger l'esprit jusqu'à le rendre capable d'énoncer des jugements solides et vrais sur tout ce qui se présente à lui * (A.T. X, 359 ; O.P. I, 77).

4. Cette nouvelle conception du sujet découle directement de la conception de la représentation scientifique que les Regulae se proposaient d'instaurer et que le Discours de la méthode présuppose. Disons brièvement, mais j'y reviendrai, que ce sujet est essentiellement le sujet "vide "de la nouvelle représentation scientifique axiomatisée de la mathesis universalis, qui constitue l'idéal gouvernant la révolution philosophique de Descartes. On peut supposer que Descartes, des Regulae jusqu'aux Méditations (et audelà), a approfondi les implications de cette nouvelle conception du sujet, qui 
Le présent texte fait le pari que l'examen des structures pragmatiques et rhétoriques qui gouvernent le "discours "qui sert de véhicule à cette légitimation philosophique du sujet permet non seulement de compléter l'analyse habituelle des concepts, mais qu'il permet d'éclairer sous un jour différent la question même de la subjectivité cartésienne et de ses processus d'instauration philosophique. Je laisserai donc de côté l'analyse de l'" ordre des raisons "pour fixer mon attention sur l" ordre du discours".

Il faudra dire d'abord quelques mots à propos du statut de la rationalité et de la méthode chez Descartes et de ce qui permet de la situer dans ce qu'il convient d'appeler le projet d' autoaffirmation" de la modernité. Dans un deuxième temps, j'analyserai brièvement l'idéal de la connaissance philosophique et la conception de la représentation qui se profilent dans les Règles pour la direction de l'esprit. Je m'arrēterai ensuite sur le Discours de la méthode, qui fournit son titre au présent texte et où ce rapport entre rhétorique et philosophie est déployé de manière "spectaculaire " - au sens propre, ce qui n'est pas étonnant de la part de l'acteur masqué des Préambules.

\section{Méthode, raison, histoire}

On l'a souvent dit, la méthode cartésienne, et de façon plus générale l'" esprit "de la philosophie cartésienne sont profondément hostiles à ce que nous appelons l'histoire. Pour le dire avec LévyBruhl, l'" autorité de la méthode ", fondée sur le modèle mathématique du savoir et son idéal de savoir certain, doit se substituer à la "méthode de l'autorité ", celle de la philosophie à laquelle Descartes s'oppose, qui repose sur un corps de doctrines fondées sur la tradition et préconisant plutōt la mémoire que la raison ${ }^{6}$. La philosophie scolastique, puisque c'est bien celle que vise Descartes, repose sur un héritage lourd et parfois contradictoire. Comme le remarque $M$. Fumaroli, le poids de cet héritage et de cette tradition "était désormais ressenti comme un obstacle pour l'inventivité de l'ingenium moderne " avec lequel la philosophie cartésienne prétend coïncider. La philosophie des écoles a besoin de fonder son autorité

apparaît clairement dans le Discours sous la forme célèbre du cogito et dans les Méditations sous la forme du ego sum dont l'essence sera saisie de plus en plus comme volonté.

5. Il faudra dire également quelques mots de conclusion sur les Méditations métaphysiques, où l'identification (et peut-ètre mème la confusion volontaire) entre le "Je * du discours et le " Je "formel, métaphysique et anhistorique qui conduit le doute est poussée au maximum à la faveur d'un genre littéraire particulier, la méditation. Mais il va de soi que l'analyse que je présente ici n'est que partielle, et qu'il serait intéressant de l'étendre non seulement aux Méditations, mais peut-être mème au Traité des passions de l'àme. Car, en dépit de ses positions anti-rhétoriques dans l'établissement de la morale, les Passions de l'áme constituent un texte qui n'est pas indifférent à sa mise en scène publique, surtout sì l'on donne crédit aux interprétations qui voient dans l'Avertissement d'un des amis de l'auteur qui sert de Préface au traité, un * masque * supplèmentaire de Descartes.

6. "The Cartesian Spirit and History ", dans Philosophy and History. Essays presented to Emst Cassirer, Oxford, Clarendon Press, 1936, p. 191-196.

7. M. Fumaroli, "La diplomatie au service de la méthode. Rhétorique et philosophie dans le Discours de la méthode ", dans La diplomatie de l'esprit De Montaigne à La Fontaine, Paris, Hermann, 1994, p. 400. 
sur la tradition, seule l'histoire peut la rendre légitime. Or, pour Descartes, c'est précisément cet inévitable élément historique qui compromet sa certitude et la confine au domaine du vraisemblable et des connaissances verbales. Seule la connaissance rationnelle peut fonder son autorité sur elle-même et sur la certitude de type mathématique des connaissances qu'elle produit, tandis que la connaissance historique est soumise à l'exercice d'une faculté inférieure de l'esprit, la mémoire, qui ne peut produire l'engendrement complet de l'ordre de ses connaissances, mais doit se contenter de l'emprunter à son objet. Finalement, l'opposition entre la connaissance méthodiquement engendree et la connaissance historique rejoint la distinction entre un savoir axiomatique et un savoir fondé sur le simple témoignage. Les Règles nous montrent que le savoir est soumis à l'ordre de la certitude et que l'autorité de la connaissance vraie ne peut s'appuyer que sur les deux opérations fondamentales de l'entendement que sont l'intuition et la déduction (capables d'évidence et d'immédiatetè) ${ }^{\theta}$.

Mais il faut aller un peu plus loin, et questionner la nature mème du rapport au passé qui s'instaure dans la méthode cartésienne. On peut, avec A. Cascardi, définir de manière générale le " projet fondateur "de la "modernité "philosophique comme une critique autoéclairée de l'histoire, qui vise à triompher des traditions du passé ${ }^{9}$. Il est aisé de remarquer que la philosophie cartésienne s'inscrit dans le sillage des tendances disons "modernisatrices" qui animent non seulement la science (et la philosophie) de l'époque, mais également les domaines politique, économique et mème esthêtique. Mais là où la pensée de Descartes est intéressante et contribue le plus à la mise en place de cette compréhension de soi de la modernité, c'est dans le fait que le modèle scientifique et philosophique qu'il contribue à mettre en place et à théoriser philosophiquement ne se contente pas de déployer le potentiel disons "émancipateur" (pour le dire avec Habermas) de la nouvelle méthode philosophique, sous la forme de transformations rationnelles et instrumentales de la nature, de la société, de l'individu; la réflexion fondationnelle du cartésianisme aboutit immédiatement à la théorisation de ce que $\mathrm{H}$. Blumenberg a appelé l'" absolutisme historique "de l'époque moderne ${ }^{10}$. L'autoposition de la méthode cartésienne vise à créer pour la pensée philosophique et scientifique des conditions qui transcendent radicalement toutes les conditions concrètes et historiques dans lesquelles lacte philosophique peut émerger et se développer. Ce que Blumenberg appelait l'" auto-affirmation " moderniste est ainsi l'indice d'un projet et d'une volonté d'auto-création de la raison, qui ne peut nullement être identifiée à une simple correction ou révision du passé. Ce qui se marque dans ce processus d'auto-affirmation moderne, avec lequel l'" esprit " cartésien coïncide si bien, ce n'est pas seulement une rupture avec le passé, la tradition, l'hêritage, mais plutôt la volonté de légitimer cette rupture par l'affirmation de la nécessité des réalisations de la nouvelle rationalité. La "méthode "se pose ainsi comme un commencement absolu, un point de départ sans

8. Cf. Règle III (A.T. X, 368-370 ; O.P. I, 87-90)

9. Subjectivité et modemité, Paris, P.U.F., 1995, p. 31.

10. Dans Die Legitimität der Neuzeit, Francfort, Suhrkamp, 1966. 
ancrage qui s'abstrait radicalement de tout point de départ naturel (ou historique, si vous préférez). Désormais, la vérité résulte de la soumission à la méthode et à l'ordre de la connaissance qu'elle impose $^{11}$.

On comprend qu'un tel projet d'auto-légitimation et d'autofondation de la raison produise de manière immédiate ce que Cascardi appelle une "crise de la relation entre la raison et l'histoire ", dans la mesure où la raison, ainsi comprise, est incapable de saisir dans sa pleine signification le processus par lequel elle est justement historiquement engendrèe. Cette polarité est déjà clairement exprimée dans les Regulae, où Descartes soutient - et je cite - " que nous ne deviendrons jamais philosophes, si nous avons lu tous les raisonnements de Platon et d'Aristote, et que nous sommes incapables de porter un jugement assuré sur les sujets qu'on nous propose ; dans ce cas, en effet, ce ne sont point des sciences que nous aurions apprises, semble-t-il, mais de l'histoire ${ }^{12}{ }^{n}$. L'originalité et la fonction essentielles du Discours de la méthode, dans ce contexte, me parait ètre d'avoir fourni une solution radicale à cette crise en affichant la volonté de commencer (ou de recommencer) entièrement en philosophie à partir de soi-même. Les premières parties du Discours de la méthode justifieront donc cette autoinstauration de la méthode rationnelle en montrant pour ainsi dire l'auto-création du " sujet "formel et potentiellement universel de la représentation mathématique, qui apparaîtra clairement sous la forme du cogito, ce "Je" vide et anhistorique dont l'identité se garantit d'elle-même dans l'acte de la pensée. Paradoxalement, c'est par un recours à l'histoire que ce sujet anhistorique sera présenté, ou plus précisément, par le récit autobiographique de l'histoire naturelle d'un esprit.

\section{La représentation sans sujet: les Règles}

Avant d'entrer directement dans le Discours, il n'est peut-être pas inutile de faire un bref détour par les Règles pour la direction de l'esprit, qui nous permettront de fixer les exigences de la méthode scientifique et d'indiquer l'idéal cartésien de la représentation. C'est dans les Règles que la réforme (ou la révolution) cartésienne des sciences et de la philosophie se révéle de la manière la plus spectaculaire, précisément parce que son instauration ne se théorise pas : Descartes y semble d'entrée de jeu installé dans la révolution épistémologique complète de la philosophie. Les Règles visent à établir une méthode dans laquelle la science est définie exclusi-

11. Entre les simples élans modernistes et la confiance technique quils engendrent, d'une part, et ce geste d'k absolutisme historique ", d'autre part, il y a la distance qui sépare le projet de Bacon et celui de Descartes. Comme l'a récemment fait remarquer Laurence Lampert (Nietzsche and Modern Times. A Study of Bacon, Descartes and Nietzsche. New Haven, Yale University Press, 1993, p. 146 ss). Descartes conserve les "buts " ou les - fins " de Bacon, en l'occurrence l'augmentation de la domination technique de la nature et le désir de reproduction d'un état de rapport d'immédiateté à la nature à travers sa maîtrise même, qui est la condition préalable du bonheur du genre humain (DM, 6) ; mais il les subordonne aux " moyens * cartésiens, c'est-à-dire à la réforme cartésienne du savoir.

12. Règle III (A.T. X, 367 ; O.P. I, 86). 
vement par la certitude. Ce renversement est illustré dès le départ par l'affirmation de l'unité des sciences, par opposition à la diversité et à la spécialisation des arts et des techniques ${ }^{13}$. Cette unité de la science est le résultat direct de l'unité de l'esprit connaissant. Dans une belle métaphore antiplatonicienne et antiaugustinienne, l'auteur des règles nous explique que cette unité de l'esprit, qu'il désigne aussi comme sagesse universelle, ne se divise pas plus dans ses diverses spécifications que la lumière du soleil ne reçoit de diversité des différents objets qu'elle éclaire. De la clarté totale de l'intuition jusqu'à l'épuisement de la lumière, d'un bout à l'autre de la chaine des sciences à venir, l'esprit connaissant ne reçoit jamais que le reflet de la lumière dont il est lui-même le foyer ${ }^{14}$. Dans cet idéal de science certaine (et il n'y a de science que certaine), l'" ordre des choses " est totalement subordonné à l'" ordre de la connaissance ${ }^{15}$ ". D'une telle science, pour l'heure, seules les mathématiques nous fournissent des exemples. Encore faut-il leur retirer le masque qui les recouvre et qui empêche de voir sous les différentes mathématiques particulières, sous les differents objets mathématiques, la mathesis universalis, cette science générale de l'ordre et de la mesure, qui ne sont pas des objets mais désignent, selon le mot de M. Fichant, "les modes selon lesquels les nombres, les figures et les autres choses dont traitent les mathématiques accèdent à la mathématicité, c'est-àdire à la certitude ${ }^{16}$ ". Les Règles instaurent ce qu'il convient d'appeler l'ordre de la représentation, celui qui correspond à l'esprit moderne visant à inscrire d'avance toute expérience dans une détermination mathématique possible, c'est-à-dire à déterminer la nature comme "objet". Or, une telle instauration est dans son essence métaphysique, comme l'a bien souligné Heidegger dans Qu'est-ce qu'une chose?. puisque définir la philosophie comme épistémologie, au sens où Descartes le fait dans les Règles, c'est bel et bien prendre position sur la nature du rapport entre l'homme et la nature, c'est-àdire sur son ètre.

Toutefois, il est facile de voir que la réflexion sur ce renversement est à peu près absente des Règles. Certes, Descartes y parle de l'esprit et de son unité, mais c'est plutôt pour faire disparaître ce " sujet " derrière sa représentation et l'ordre qu'elle institue. Pour qui un tel ordre de la représentation fait-il sens et comment est-il instauré ? Ce sont des questions qui ne sont pas abordées par les Règles, qui ne traitent de ce que l'on appelle la "subjectivité "qu'en filigrane, ou plutôt en creux, puisque l'ordre mathématique de la représentation est pensé comme possédant une sorte d'autarcie. Le " sujet " de la science nouvelle se pose lui-mème en mème temps que ce nouvel ordre épistémique assure son auto-position, mais il n'est

13. C'est l'objet de la Règle I.

14. Règle I (A.T. X, 360 ; O.P. I, 78). À ce sujet, on lira le texte éclairant - si je puis dire - de E. Cassirer : "Descartes et l'unité de la science", in Revue de Synthèse, XIV, 7, 1937.

15. On consultera avec profit la magnifique reconstruction que propose JeanLuc Marion de ce problème anti-aristotélicien: Sur l'ontologie grise de Descartes, Paris, Vrin, 1975 ( $2^{\mathrm{e}}$ éd., 1993), notamment le "Chapitre premier : l'universalité de la science unique ", p. 25-69.

16. "Les fondements de la science moderne", dans Magazine Littéraire, avril 1996 , p. 56. 
pas disponible dans cet acte d'auto-affirmation, et ce pour une raison essentielle : c'est que cette représentation est pensée comme emportant sa propre évidence et comme entièrement transparente à elleméme. Et pourtant cette subjectivité reste présente comme entité implicite et schématique dans l'ordre de la représentation ; le "sujet "des Règles n'est pas défini par son "contenu "(en tant qu'entité possédant une identité narrative, historique, autobiographique), mais en tant que condition de possibilité d'un discours axiomatique qui affirme son propre pouvoir de représenter ${ }^{17}$. Ce sujet gouverne l'ordre de la représentation, comme s'il existait en dehors de la représentation. On pourrait ajouter qu'il le gouverne, pour l'instant, sans le garantir, ce qui sera la tâche ultérieure du Discours et des Méditations. Disons pour conclure ce point que les règles définies par Descartes préfigurent ou contiennent l'auto-position de l'homme comme " sujet "; elles contribuent à rendre pensable le point de vue selon lequel - ou à renforcer l'illusion selon laquelle - on peut tout recommencer ex nihilo, et que l'homme comme "sujet " est en mesure de se projeter lui-mème en dehors de toute historicité et de toute tradition afin de créer un nouveau langage axiomatique, qui serait l'expression de la "raison". En pensant l'épistémologie nou-

17. À ce sujet, voir Dalia Judovitz, Subjectivity and Representation in Descartes. The Origins of Modernity. Cambridge, Cambridge University Press, p. 84. Les analyses de $D$. Judovitz permettent également de mettre en contraste la conception cartésienne de la représentation avec la conception qu'un auteur comme Montaigne se faisait de la représentation, du savoir et du langage. Comme elle l'a suggéré, cette fondation cartésienne de lordre de la représentation repose sur la présupposition de la transparence de la représentation. Cette transparence lui est conférée par la certitude interne des mathématiques qui constituent son paradigme épistémique. Chez Montaigne, à l'inverse, l'accès au "moi * est inséparable des processus narratifs de présentation et de représentation de ce "moi "dans le discours. Jamais une représentation du " moi " ne pourra produire un savoir entièrement validé de soi, comme le " sujet " philosophique cartésien - le cogito du Discours et l'ego sum des Méditations - prétendra le faire. Il n'y a de rapport à soi que de manière médiate et indirecte, par le biais d'une représentation qui crée un rapport à soi-même et une sorte "d'étrangeté ". En général, le XVI $I^{c}$ siècle et le début du XVII siècle - que l'on peut appeler par commodité le "baroque ", et dont Montaigne, sur ce point, est un bon représentant - est animé d'une conscience de l'opacité des processus représentatifs. L'histoire et la tradition ne constituent plus un réservoir de normes absolues et d' exemplarité " cognitive et morale, mais pour autant, l'idéal d'un ordre transparent de la représentation, correspondant à l'expression de la raison dont l'homme serait le " sujet ", n'a pas encore fait son apparition. Au contraire, la conscience de la séparation entre les mots et les choses ("Nous n'avons aucune commurication à l'ètre ", nous dit Montaigne) conduit à la reconnaissance des diverses stratégies discursives de définition de l'identité et à la relative autonomie des divers systèmes de représentation. Le savoir apparait conséquemment sous la forme de la traduction et de l'interprétation, plutót que sous la forme d'un système fixe d'évidences démonstratives: " nous ne faisons, dit Montaigne, que nous entreglosser * (Essais, III, 13). De manière analogue, le rapport à soi-méme ne conduit nullement à la domination rationnelle de notre essence fixe comme pensée et auto-rèflexion, mais au contraire à la prolifération de notre identité. On voit immédiatement les conséquences " sceptiques " de cette conception de la représentation, et c'est donc un usage complètement diffërent de l'autobiographie que devra faire le Discours pour justifier l'adhésion du lecteur au " sujet " garantissant l'ordre nouveau de la représentation scientifique. Voir Judovitz, op. cit., " 1 : From Self to Subject : Montaigne to Descartes ", p. 8-38. 
velle comme devant rompre radicalement avec les traditions qui la précèdent afin de se produire comme discours formel autonome fondé sur des instances anhistoriques comme l'évidence, l'intuition, la déduction, l'énumération, Descartes prépare la fusion de la représentation et du sujet de la représentation qui constitue la vision cartésienne de l'Homme moderne. Ce sera ensuite sur la confusion entre ce sujet formel et le sujet empirique que la force pragmatique de la fondation cartésienne de la subjectivité pourra s'appuyer dans le Discours et les Méditations.

\section{Subjectivité et narrativité dans le Discours de la méthode}

Il sera impossible ici d'entrer dans le détail des stratégies pragmatiques et rhétoriques mises en œuvre dans le Discours de la méthode, aussi devrai-je me contenter de souligner les principaux rapports entre la rhétorique et la philosophie dans le Discours. Il convient de souligner d'entrée de jeu, grâce à quelques exemples, le caractère paradoxal du Discours. L'auto-présentation autobiographique entre en opposition avec le "sujet philosophique neutre" qu'elle est censée instituer : l'" histoire d'un esprit "vise à mettre en scène un sujet rationnel radicalement "anhistorique ": l'" hétéronomie "de l'enfance et de l'esprit soumis aux préjugés de son histoire et de ses désirs est distincte de l'" autonomie "philosophique : la "représentation " quasi-montaignienne de soi se heurte à la "transparence " de la représentation issue de la conception mathématique du savoir dont la seconde partie du Discours nous livre les "préceptes" de base ; l' exemplarité "mimétique de la fable est incompatible avec l'autorité auto-fondée de la raison philosophique.

Il n'est peut-être pas inutile de rappeler sommairement le plan de cet ouvrage célèbre. Dans la première partie, Descartes raconte les attentes et les désillusions suscitées par sa formation au collège jésuite de LaFlèche, ainsi que la période de ses voyages et de son engagement dans diverses armées. Descartes est déçu tout autant par le "livre du monde " que par les " livres des Anciens". Le rôle de ces années de jeunesse semble avoir été plutôt cathartique, et Descartes en tire l'unique résolution de se dégager de la coutume et de l'exemple pour "étudier en lui-même", nous dit-il, et "employer toutes les forces de son esprit à choisir les chemins qu'il devait suivre $^{18}$ ". La seconde partie raconte le célèbre épisode du " poēle " de novembre 1619, où Descartes eut l'intuition de sa philosophie à venir. Il y fait retour sur son expérience passée, et tirant les leçons des événements où sa vie eut "l'heur" de le mettre, il élabore une réflexion sur l'unité de la science et sur une logique nouvelle tirée de la manière de pensée des mathématiques, qui avaient constitué le seul ilot de satisfaction de sa formation intellectuelle, en raison de la certitude dont elles se montraient capables. Il y avait sans doute dans cette nouvelle "logique ", qui est une logique de la découverte et non, comme la logique scolastique, une logique de l'exposition, le fondement d'une grande réforme de la manière de pensée philosophique et scientifique. Cette manière de pensée, cette "méthode", que Descartes ne souhaite pas généraliser à tous mais se contente de 
s'appliquer à soi-même, pourra répondre à l'interrogation initiale et naturelle de Descartes, qui tendait spontanément à rechercher à conduire rigoureusement sa vi $\mathrm{e}^{19}$. L'auto-présentation de ses années de collège place dans ce désir la source mème du mécontentement de Descartes face à son éducation : il semble y avoir toujours antinomie entre la richesse des réponses pratiques promises par une discipline et la rigueur de la connaissance qu'elle livre. Il serait intéressant de voir que Descartes, dans ce contexte, oppose la rigueur vide des joutes dialectiques métaphysiques à la pure persuasion rhétorique de la morale, comme il oppose symétriquement les mathématiques particulières, productrices de certitudes mais confinèes à des domaines objectifs spécifiques, à la physique aristotélicienne, qui prend pour objet la nature en général, mais reste purement qualitative et par conséquent complètement dépourvue de rigueur ${ }^{20}$. La troisième partie du Discours présente ce que Descartes appelle sa morale "par provision", en attendant une morale "ajustée à la raison ". La quatrième pose le résultat de la réflexion métaphysique de Descartes sur la nature de notre connaissance certaine. C'est dans ce contexte que le "Je pense, donc je suis "apparaît comme la première certitude et comme le critère de toute connaissance certaine qui pourra ètre établie. Les Méditations iront beaucoup plus loin en jouant des thèmes mèmes de la littérature "baroque " et néosceptique et en posant, par le biais du doute hyperbolique (et pas seulement méthodique) et grâce à l'artifice du " mauvais génie ", la question de la validité objective de la représentation, c'est-à-dire remettra en question la représentation elle-mème et, en allant jusqu'à questionner la certitude mathématique, modèle de toute clarté, elle remettra en question la rationalité elle-mème. La cinquième partie se veut un échantillon de la science cartésienne et de l'application de la méthode cartésienne, tirées des traités $D u$ monde et $D e$ l'homme restés non publiés ${ }^{21}$. La sixième partie revient sur les implications techniques et morales de la nouvelle méthode et sur les raisons qui ont conduit Descartes à sortir de son silence relatif et à rendre publique sa pensée ${ }^{22}$.

19. On se souviendra que les Obsenvations du jeune Descartes font état dune citation d'Ausone, qui se retrouvera dans les réves relatés par les Olympiques: "Quelle voie suivrai-je en la vie?" (Quod vitae sectabor iter?). Cf. O.P. I, 49.

20. On peut toutefois croire qu'il s'agit là, dans une large mesure, d'une illusion rétrospective, voire d'une réécriture rhétorique de son expérience passée. mais la question est complexe, puisque, comme j'ai eu l'occasion de le souligner, l'importance du thème de l'orientation pratique de la pensée et de la conduite pratique de la vie est enracinée dans une disposition d'esprit précoce, qui est restée constante par la suite.

21. Descartes y présente l'un des plus beaux fleurons de sa méthode dans le domaine de la médecine, à ses yeux, son explication mécanique de la circulation du sang. Il complète ces remarques par des observations sur ce qu'il conviendrait d'appeler une psychophysiologie, qui témoigne d'une volonté de saisir les rapports entre l'esprit et le corps, qui s'appuie sur un dualisme métaphysique que le détour par la métaphysique permettait de clarifier et sur lequel les Méditations reviendront de manière plus précise.

22. Cette dernière partie achève le parcours pragmatique du Discours, puisqu'elle explicite non seulement les espérances de Descartes par rapport à sa méthode. mais également les liens complexes et peut-ètre contradictoires que Descartes souhaite établir avec les destinataires de son 
Dès le départ, le Discours de la méthode se présente comme une sorte de "contrat " entre l'auteur et son public. Une certaine fusion des rôles et des identités est essentielle pour que l'exemplarité d'un discours puisse fonctionner. On apprend ainsi que le "bon sens est la chose du monde la mieux partagée ${ }^{23}$ ". On y a vu souvent une marque d'ironie qui n'est certainement pas complètement absente, mais l'essentiel ici est que Descartes insiste d'entrée de jeu sur ce que l'auteur et son public recherché ont en commun. Ce public qui peut revendiquer pour lui-mème ce pouvoir de bien juger que l'on appelle le "bon sens" ou la raison, c'est ce public des "honnètes gens " qui s'étend désormais, au-delà des doctes, à l'ensemble des gens capables d'utiliser leur raison, "ces gentilshommes de cour, ces bourgeois et artisans des villes "24, qui ont l'avantage d'avoir l'esprit moins embarrassé d'autorités traditionnelles, et sont donc plus susceptibles d'être sensibles à cet appel de l'auteur. Descartes en appelle ici à la distinction scolastique entre la forme et l'accident pour justifier cette présence intégrale de la raison comme forme en chaque individu, l'accident étant ce qui différencie les membres d'une même espèce ${ }^{25}$. Après avoir produit cette captation d'attention et cette identification, Descartes doit se dégager du lecteur pour marquer sa différence et fonder sa légitimitè à écrire. Il présente alors immédiatement sa vie comme une sorte daccident qui le singularise. Cet "accident " (qui est à prendre également au sens courant du terme) l'autorise à présenter l'histoire de son esprit comme une suite d'événements qui l'ont conduit à élaborer la méthode d'une science nouvelle à portée universelle. Je "pense, dit Descartes, avoir eu beaucoup d'heur, de m'ètre rencontré dès ma jeunesse en certains chemins, qui mont conduit à des considérations et des maximes, dont j'ai formé une méthode, par laquelle il me semble que j'ai moyen d'augmenter par degrés ma connaissance, et de l'élever peu à peu au plus haut point, auquel la médiocrité de mon esprit et la courte durée de ma vie lui pourront permettre d'atteindre ${ }^{26}$. La différence qui

Discours, ce "public "composè de tous les "honnêtes gens "auxquels il s'adressait dès l'ouverture en faisant appel a l'universalité du "bon sens ": Descartes semble contribuer à constituer une nouvelle "communauté scientifique " en court-circuitant ses pairs de la "République des Lettres ", en créant une sorte d'ethos de coopération bienveillante entre esprits autonomes, en mème temps qu'il cherche à lutter contre le dessaisissement de son message et qu'il affirme vouloir veiller lui-mème aussi bien à l'interprétation de sa pensée qu'à la gestion des expérimentations pour lesquelles il sollicite des fonds. C'est pourquoi A. Philonenko, récemment, a pu présenter avec humour le Discours de la méthode let les essais qui laccompagnent) comme une sorte de demande de subvention auprès d'un public élargi, sensible aux applications techniques de la méthode (Relire Descartes, Paris, J. Grancher, 1994). Toutefois, lorsqu'on lit cette section du Discours, on voit à quel point cette atmosphère de collaboration créée par la rhétorique mēme du texte est ambiguë et éloignée de la disposition d'esprit de Descartes lui-même (plutōt préoccupé de tranquillité et souhaitant, en même temps, "contröler "le sens de ses écrits), et on comprend que la "maison de Salomon "cartésienne est éloignée de la préfiguration d'un grand C.N.R.S. ou mēme de l'utopie baconienne.

23. A.T. VI, 1 ; O.P. I, 568 .

24. Selon l'expression de Fumaroli, op. cit., p. 379-380.

25. A.T. VI, 2-3; O.P. I, 568-569.

26. A.T. VI, 3 ; O.P. I, 570 . 
autorise l'auteur à écrire n'est pas une raison plus parfaite, mais une raison mieux conduite, c'est-à-dire une juste méthode.

Il faut souligner ce qu'on peut appeler l'exemplarité problématique du Discours de la méthode. Descartes se propose de montrer les chemins qu'il a suivis et "de représenter [sa] vie comme en un tableau $^{27}$ "et non pas d'enseigner sa méthode. En fait, la méthode ne s'enseigne pas, même si elle peut s'apprendre et surtout, s'exercer. Mème les supposées "règles "ènoncées dans la deuxième partie du Discours (l'évidence, la division, la déduction et l'énumération) ne sont que des " préceptes "qui ne peuvent dicter en mème temps les conditions de leur application. Plutôt que d'enseigner la méthode, Descartes se propose de montrer comment il a tâché de conduire sa raison; l'autorité de cette méthode vient de la raison elle-même, et non pas de l'individu Descartes qui avoue ne pas vouloir assumer cette position d'autorité didactique. C'est donc une histoire, ou "si vous l'aimez mieux", une fable qui nous est proposée, la fable que constitue l'histoire de sa propre pensée. Mais c'est une fable qui renonce aussitôt à la majeure partie de son potentiel didactique, puisqu'elle constitue pour ainsi dire un "exemple inimitable ", ou qu'il ne convient pas d'imiter. Et ce pour deux raisons. Premièrement, parce que l'histoire en question est purement naturelle, " accidentelle ", et qu'elle n'a de valeur et n'obtient d'autorité que par son aboutissement, c'est-à-dire par l'émergence d'un individu capable de penser par soi-même, dépositaire de cette "subjectivité philosophique "que le Discours met au jour pour la premiere fois. Descartes crée ainsi, suivant la belle formule de M. Fumaroli, "un mythe platonicien énoncé à la première personne ${ }^{28}$ ", celui de la naissance, ou de la re-naissance d'une autonomie individuelle. Or, la fable du Discours ne peut ètre didactique puisqu'on ne peut pas expliquer l'émergence de la rationalité telle que la pense Descartes, dans sa dimension d' absolutisme historique ". En fait, le discours autobiographique fonctionne plutôt par sa dimension d'authenticité que par sa fonction d'exemplarité normative, et par la confusion qu'elle établit entre le sujet philosophique de la rationalité et le sujet autobiographique du Discours; il vise moins à ètre imité qu'à servir de légitimation à l'auto-création du sujet de la connaissance certaine. La fonction pragmatique du Discours, dans ce cas, serait de tabler au maximum sur la confusion entre lauto-création du sujet personnel, empirique, autobiographique, d'une part, et cette dimension "autoaffirmative " de la nouvelle rationalité, d'autre part.

La seconde raison du renoncement à la dimension didactique de la fable autobiographique vient de la crise mème de l'exemplarité et de l'autorité historique que la pensée cartésienne pousse à son maximum. On a vu à quel point l'esprit cartésien était hostile à l'histoire. Cette méfiance s'étend à toute forme d'exemplarité non fondée sur la certitude rationnelle. Descartes accentue la crise de l'exemplarité entamée à la fin de la Renaissance, non pas pour aboutir à un scepticisme montaignien, mais pour faire apparaître la légitimité d'une subjectivité formelle, neutre et potentiellement 
universelle. Comme cette légitimation ne peut être pensée qu'en tant qu'autolégitimation, on ne pourra penser aucun passage historique et naturel de l'hétéronomie à l'autonomie ${ }^{29}$. Dès lors, la plupart des gestes significatifs du sujet autobiographique du Discours auront une valeur négative, et consisteront à suspendre l'adhésion aux normes exemplaires déterminées par l'autorité historique ou par la coutume. Ainsi en va-t-il du rapport globalement négatif de Descartes à la lecture et au voyage. L'autorité du texte écrit est dangereuse, puisqu'elle repose sur la persuasion plutôt que sur la conscience de soi. L'autorité de la coutume est suspecte du fait mème de la diversité des mœurs. Celui qui lit trop, comme celui qui voyage trop, risque de devenir étranger à lui-mème. Descartes exacerbe la crise de confiance dans la normativité des modèles du passé, qui avait déjà été soulignée par des écrivains comme Cervantes. L'autorité du texte écrit n'est plus une valeur, mais un danger, le danger de donner au lecteur des illusions à propos de sa propre nature ${ }^{30}$. L'exemplarité du Discours est donc plutòt négative ou mème cathartique : en produisant la négation des expériences d'identification négative, elle enjoint au lecteur "d'étudier en lui-même " et d'assumer lui-mème la tâche de savoir comment utiliser les exemples, ce qui est à tout le moins la condition préalable de l'autonomie.

On peut remarquer, avec $J$. D. Lyons, qu'il y a trois sens distincts du "Je "- ou du "sujet " - dans le Discours" ${ }^{31}$. On pourrait dire trois " masques ". D'abord, le sujet autobiographique, c'est-à-dire l'individu Descartes qui se prèsente comme se "créant "lui-même comme "Je ", par le rejet de tout exemple préalable. Deuxièmement, le sujet éthique, qui est un sujet clivé entre une figure externe sociale et une face intérieure qui est consacrée à la tâche de réformer sa pensée. Ce " je " éthique dont la première maxime est la modération est le masque social de la prudence, qui est une dimension essentielle de la conception cartésienne de l'individu. Jamais Descartes ne présente la réforme philosophique de la pensée comme un modèle à suivre, et la "résolution d'entreprendre soi-mème de se conduire " ne vaut que pour lui-même. Il partage les mêmes réticences que Montaigne envers les "réformateurs " de ces grands corps que sont les institutions comme l'État, la science ou la religion ${ }^{32}$. Descartes peut être vu comme un "législateur prudent ${ }^{33}$ "qui peut en même temps présenter son " exemple " personnel et s'en dégager en se rabattant sur ce que j'ai appelé son identité autobiographique, puisque l'autorité qui fait de son cheminement un "modèle "lui est procurée non pas par sa vie, mais par la raison elle-même. Caché derrière le "masque "de la

29. Ou, pour le dire avec Kant, de la minorité à la majorité philosophique.

30. Pensant peut-être au Don Quichotte, Descartes mentionne ainsi que "ceux qui règlent leurs mœurs par les exemples qu'ils tirent [des histoires et des fables] sont sujets à tomber dans les extravagances des paladins de nos romans, et à concevoir des desseins qui passent leurs forces " (A.T. VI, 7 ; O.P. I, 574).

31. "Subjectivity and imitation in the Discours de la méthode", dans Neophilologus, vol. 66,1982 , p. 516 ss.

32. Cf. la deuxième partie du Discours (A.T. VI, 13-15; O.P., 581-582) et les rêférences précises d'É. Gilson aux textes des Essais de Montaigne et de De la sagesse de $\mathrm{P}$. Charron, $\hat{\mathrm{E}}$. Gilson, Discours de la méthode, texte et commentaire, Paris, Vrin, 1939, p. 173-174.

33. L'expression est de L. Lampert, op.cit., p. 143 ss. 
modération, on ne pourra pas voir en lui le révolutionnaire potentiellement dangereux qu'y chercheront ceux qui, au siècle suivant, verront dans la Révolution française l'aboutissement de la révolution cartésienne. Sur le plan disons "politique ", Descartes tient à séparer nettement les genres : la "raison " (et la " subjectivité " philosophique qui en est le dépositaire) commande dans son royaume, qui est celui de la représentation scientifique, tout comme le Roi est seul législateur en matière de lois et d'institutions politiques ${ }^{34}$. et comme les institutions religieuses reposent sur l'autorité de la Révélation, dont la nouvelle " raison " n'entend pas encore contester l'interprétation aux Théologiens. La morale par provision sert à " bien vivre " en l'absence de raisons absolument déterminantes, c'est-àdire lorsqu'il n'est pas possible, comme le souhaite Descartes depuis le début, "de bien juger pour bien faire". Mais n'oublions pas que pour "bien vivre", il faut aussi "bien se cacher". Le sujet éthique. tout comme la morale qu'il rend possible, est un sujet "par provision $^{35}$ ".

La troisième figure importante du " Je " qui est développée dans le Discours est le sujet métaphysique ou le cogito, véritable terme de la présentation narrative, et point de départ authentique de la chaine des vérités philosophiques. Le "Je pense, donc je suis " est une vérité si ferme et si assurée, nous dit Descartes, "que je jugeai que je pouvais la recevoir, sans scrupule, pour le premier principe de la philosophie que je cherchais ${ }^{36}$ ". Je passe ici sur les ambivalences des formulations du cogito dans le Discours, ambivalences qui seront à mon sens en grande partie levées dans les Méditations, pour me concentrer sur la narrativité du texte cartésien. On peut reconnaitre, avec J. Lyons ${ }^{37}$, que le principal effet rhètorique du cogito lui est conféré par la position même qu'il occupe dans le Discours de la

34. C'est ce qu'affirmait déjà la lettre à Mersenne du 15 avril 1630, à la faveur d'une analogie entre l'institution divine des lois de la nature et l'institution des lois politiques, dans le contexte d'une réflexion sur la "création des vérités éternelles ": " Ne craignez point, je vous prie, d'assurer et de publier partout que c'est Dieu qui a établi ces lois en la nature, ainsi qu'un roi établit des lois en son royaume "(O.P. I, 260). En général, dans le Discours, cette "réserve " de la nouvelle méthode n'est pas sans entrer en conflit potentiel avec ses exigences et ses prétentions premières. Descartes, qui en est parfaitement conscient, semble éprouver le besoin de se prémunir d'avance contre d'éventuelles accusations d'irréligion ou de "réformisme " politique. Le problème est moins délicat dans le domaine théologique. où ce n'est pas essentiellement par conformisme ou par conservatisme que Descartes prône la fidélité à la "religion de sa nourrice", mais pour des raisons philosophiques, parce que les "vérités " révélées dépendent justement d'une lumière surnaturelle : d'où, par ailleurs, l'impossibilité, pour Descartes, de comprendre et de justifier la " conversion " religieuse du point de vue rationnel. Le problème est plus épineux lorsqu'on en vient à la question politique, étant donné l'utilisation que fait Descartes lui-mème, dans la seconde partie du Discours. de l'analogie entre l'unité d'intention rationnelle qui garantit la valeur d'un projet technique (architectural, par exemple) et d'une constitution (Descartes cite l'exemple de Sparte), et l'unité de l'esprit et de la science, qui caractérise la réforme de la pensée philosophique qu'il préconise.

35. Je laisse ici de côtê le problème de l'évolution de cette morale "provisoire * vers une morale que l'on dit " définitive "

36. Discours, IV (A.T. VI, 32 ; O.P. I, 603).

37. Op. cit., p. 520 . 
méthode. Il intervient à peu de choses près "au milieu "du Discours ; il est à la fois l'aboutissement de ce qui précède et le point d'inauguration de toute une nouvelle série de remarques fondées sur la vérité. Véritable " sommet "du Discours, il est le lieu du passage de l'erreur à la vérité. Dans les Méditations, c'est la preuve de l'existence de Dieu et de sa véracité qui occupera cette position transitoire : la véracité divine, en rétablissant la validité objective de l'ordre représentatif, permet d'entamer ce qu'on peut appeler la phase " descendante " par laquelle le sujet de la connaissance est fondé à " reconstruire "les vérités et la science qu'il avait détruites lors du doute hyperbolique.

J'ai suggéré précédemment que cette narrativitè du Discours comme d'ailleurs des Méditations - ne joue pas un rōle simplement ormemental dans les textes cartésiens, mais qu'elle contribue fortement à la légitimation du point de vue philosophique avancé par Descartes. On peut suivre ici encore l'interprétation de Lyons dans ses grandes lignes, en soulignant les deux manières dont le sujet "plein" du discours autobiographique préfigure et soutient le sujet "vide" (ou neutre) de la vérite ${ }^{38}$. Premièrement, on peut remarquer que la perspective du cogito est préparée par le processus de négation existentielle et spontanée des modèles d'imitation annoncé par les deux premières parties du Discours, qui mettent directement en scène le sujet que j'ai appelé autobiographique. Le cogito répète de manière systématique ces diverses négations sur le plan philosophique. Le sujet philosophique peut bien se "vider "de tout, sauf précisément de la pensée qui le définit; il se débarrasse de tout contenu pour se poser comme point de départ absolu de tout savoir certain et comme fondement de la représentation. Deuxièmement, on peut voir que le "Je "autobiographique est là essentiellement pour créer l'impression d'un "Je "métaphysique plein, qui puisse, en vertu de cette confusion, partager notre "monde". Le "Je" empirique foumit au "Je" de la vérité l'illusion d'un support. Pourtant, les différences sont importantes entre les deux "sujets " et l'illusion pragmatique ne saurait les effacer ; en effet, la certitude du cogito est issue soit d'une performance de la pensée, soit d'une inférence nécessaire et immédiate (je ne tiens pas à discuter cette question pour elle-méme), mais en tous cas elle ne peut pas résulter d'une " narration ", dans la mesure où une telle vérité présuppose le rejet préalable de toute extériorité, et par le fait mème de tout modèle. L'autorité sur laquelle se fonde la certitude première qu'est le cogito est empruntée à la raison elle-mēme ; elle est par conséquent au-delà de l'" histoire".

D. Judovitz ${ }^{39}$ a fait pertinemment remarquer que c'est tout le discours autobiographique qui préfigure et soutient le cogito par son caractère "performatif ". De ce point de vue, la " performance "qu'est la saisie indubitable de l'acte réflexif de la pensée serait annoncée par le caractère performatif du discours autobiographique ; en mème temps qu'il décrit les événements de son cheminement intellectuel, le sujet empirique du Discours de la méthode accomplit quelque chose :

38. Ibid.

39. Op. cit., p. 133. 
il fait correspondre, comme j'ai eu l'occasion de le souligner, l'autocréation du sujet autobiographique et l'auto-création du sujet rationnel. Loin d'ètre l'erreur que Husserl y voyait, l' illusion "qui nait de la confusion du sujet empirique et du sujet transcendantal pourrait bien être l'une des clés de la force et du succès historique du discours philosophique cartésien. Descartes compense ainsi l'oubli de la dimension narrative ou historique de l'identité du "soi " qui est présupposée par le cogito par le recours à des procédés rhètoriques complexes qui emportent le lecteur, le sujet empirique et le sujet métaphysique dans un mouvement d'identification qui permet de penser et de médiatiser l'idéal nouveau de la subjectivité. Dans le cas du Discours, l'autobiographie devient un médium puissant pour la personnification du sujet de la vérité.

Je ne peux terminer ce texte sans ouvrir la perspective sur les Méditations métaphysiques, qui devraient faire l'objet d'une analyse analogue à celle que je viens de fournir du Discours de la méthode. Les Méditations partent du "sujet philosophique" - le cogito du Discours - et visent à répondre à la question du fondement ontologique de nos représentations. Elles développent une autre stratégie narrative, en recourant à un genre littéraire déterminé, la "méditation", où, contrairement à ce qui se passe dans louvrage de 1637, le spectateur et l'acteur se confondent à toutes fins pratiques dans l'exercice de la méditation. Le genre "méditatif "adopté par Descartes fait immédiatement vérifier à chaque lecteur, sous la forme d'une " expérience ", l'enchainement en principe " neutre " de l'" ordre des raisons" métaphysiques gouverné par le mode analytique d'exposition des certitudes. Gráce à cette identification parfaite entre le lecteur et le narrateur dans l'espace de la méditation, c'est chaque sujet empirique procédant à cette substitution des rôles et devenant " sujet méditant " qui peut désormais servir de support sensible et de personnification au sujet métaphysique. Mème l'insistance du sujet méditant narrateur sur tous les détails sensibles (son corps, ses vêtements, les lieux) ne semble faite que pour favoriser l'identification de chaque lecteur avec sa réalité concrète et pour faciliter paradoxalement l'expérience de négation des représentations sensibles sans laquelle l'expérience métaphysique est impensable. Indépendamment des différences philosophiques profondes entre le Discours et les Méditations, et compte tenu de la diversité des moyens rhétoriques mis en œuvre dans chacun de ces textes, il y a entre les deux quelque chose de semblable, et c'est la nécessité de compenser l'aspect "vide " du sujet métaphysique par l'illusionnisme ou le trompe-l'œil des procédés narratifs. Dans ce sens du moins, il est possible de dire que Descartes met le "baroque" au service du "classicisme ".

Département de philosophie Université de Montréal 